\title{
Treatment of Gilles de la Tourette Syndrome with Cannabis-Based Medicine: Results from a Retrospective Analysis and Online Survey
}

\author{
Leonie M. Milosev, ${ }^{1}$ Nikolas Psathakis, ${ }^{1}$ Natalia Szejko, ${ }^{2,3}$ Ewgeni Jakubovski, ${ }^{1}$ and Kirsten R. Müller-Vahll, ${ }^{1, *}$
}

\begin{abstract}
Introduction: Gilles de la Tourette syndrome (GTS) is a neuropsychiatric disorder that is characterized by motor and vocal tics and psychiatric comorbidities, including attention deficit/hyperactivity disorder (ADHD) and obsessive-compulsive behavior/disorder (OCB/OCD). From anecdotal reports and preliminary controlled studies, it is suggested that cannabis-based medicine (CBM) may improve tics and comorbidities in adults with GTS. This study was designed to further investigate efficacy and safety of CBM in GTS and specifically compare effects of different CBM.

Materials and Methods: First, we performed a retrospective data analysis including all those adult patients seen at our clinic, who had used CBM for the treatment of GTS at some time. All these patients were asked to complete an online survey (second study part) to receive more detailed data about treatment with CBM.

Results: From medical records, we identified 98 patients who had used CBM (most often street cannabis followed by nabiximols, dronabinol, medicinal cannabis) for the treatment of GTS: Of the 38 patients who were able to judge, 66\% preferred treatment with medicinal cannabis, 18\% dronabinol, 11\% nabiximols, and 5\% street cannabis. Altogether, CBM resulted in a subjective improvement of tics (of about $60 \%$ in $85 \%$ of treated cases), comorbidities (55\% of treated cases, most often OCB/OCD, ADHD, and sleeping disorders), and quality of life (93\%). The effects of CBM appear to persist in the long term. Adverse events occurred in half of the patients, but they were rated as tolerable. Dosages of all CBM varied markedly. Patients assessed cannabis (with a preference for tetrahydrocannabinol [THC]-rich strains) as more effective and better tolerated compared with nabiximols and dronabinol. These data were confirmed by results obtained from the online survey $(n=40)$.

Conclusion: From our results, it is further supported that CBM might be effective and safe in the treatment of tics and comorbidities at least in a subgroup of adult patients with GTS. In our sample, patients favored THC-rich cannabis over dronabinol and nabiximols, which might be related to the entourage effect of cannabis. However, several limitations of the study have to be taken into considerations such as the open uncontrolled design and the retrospective data analysis.
\end{abstract}

Keywords: cannabis; cannabis-based medicine; Gilles de la Tourette syndrome; tics

\section{Introduction}

Gilles de la Tourette syndrome (GTS) is a neuropsychiatric disorder that is characterized by motor and vocal tics. It is often associated with psychiatric comorbidities such as attention deficit/hyperactivity disorder (ADHD), obsessive-compulsive behavior/disorder
(OCB/OCD), anxiety, rage attacks, sleeping disorders, and depression. ${ }^{1}$ Pathophysiologically, most evidence supports a "dopaminergic hypothesis" of GTS manifested by an abnormal interaction between tonic and phasic dopaminergic signaling, resulting in altered modulation of cortico-striato-thalamo-cortical circuitry. ${ }^{2}$ Accordingly,

${ }^{1}$ Clinic of Psychiatry, Social Psychiatry and Psychotherapy, Hannover Medical School, Hannover, Germany.

Departments of ${ }^{2}$ Neurology and ${ }^{3}$ Bioethics, Medical University of Warsaw, Warszawa, Poland.

*Address correspondence to: Kirsten R. Müller-Vahl, MD, Clinic of Psychiatry, Social Psychiatry and Psychotherapy, Hannover Medical School, Carl-Neuberg-Straße 1, 30625 Hannover, Germany, E-mail: mueller-vahl.kirsten@mh-hannover.de 
antipsychotics are the most widely used drugs for the treatment of tics. ${ }^{3,4}$ However, due to frequently associated adverse events (AEs) and insufficient efficacy, many patients are dissatisfied and, therefore, seek for alternatives, including self-treatment with cannabis. ${ }^{5,6}$ According to surveys from Germany and Canada, street cannabis leads to a tic reduction of about $60 \%$ in more than $80 \%$ of patients. ${ }^{5,6}$ Remarkably, several patients report, in addition, an improvement of psychiatric comorbidities including $\mathrm{ADHD}$, OCD, anxiety, and rage attacks. Most often reported AEs were a feeling of being "high," decreased concentration, increased anxiety, increased appetite, sedation, irritability, and dry mouth and eyes. ${ }^{5,6}$

Until today, only two small randomized controlled trials (RCT) have been carried out investigating the efficacy and safety of dronabinol (tetrahydrocannabinol, THC) in GTS. ${ }^{7,8}$ In both studies, dronabinol was superior to placebo and resulted in a significant tic reduction. The most often reported AEs were tiredness, dry mouth, dizziness, and muzziness. Only recently, single case studies have been published reporting on beneficial effects similar to street cannabis and dronabinol after use of the cannabis extract nabiximols (containing $2.7 \mathrm{mg}$ THC and $2.5 \mathrm{mg}$ cannabidiol [CBD] per puff) ${ }^{9,10}$ and medicinal cannabis (THC-rich strains), ${ }^{11,12}$ respectively.

Thus, from available data ${ }^{5-18}$ it is suggested that cannabis-based medicine (CBM) is effective and well tolerated in the treatment of GTS and improves tics as well as a wide range of comorbidities. It is unclear, however, whether different CBM and routes of intake differ in efficacy and safety. With respect to longterm treatment effects of cannabinoids, so far only limited data obtained from open uncontrolled case studies are available. ${ }^{6,11}$ This study was designed to increase our knowledge about the effectiveness and safety of CBM in the treatment of GTS, particularly with respect to differences between different CBM.

\section{Materials and Methods}

This study consisted of two parts: In the first part, we included all those outpatient subjects with GTS, who were seen at our Tourette outpatient clinic at Hannover Medical School at least once between 2002 and 2017. The following inclusion criteria were established: (1) age $\geq 18$ years and (2) CBM (either self-medication with street cannabis or CBM prescribed by any medical doctor) for the treatment of GTS at some time. The exclusion criteria were: (1) age $<18$ years and (2) recreational use of cannabis. All patients included in the first part were asked to participate in the second part. The study was approved by the local ethics committee. All patients gave written informed consent before entering in the second part of the study.

\section{Part 1: retrospective data analysis}

About 1000 records of GTS patients were screened (01-04/2017) and-if inclusion criteria were fulfilledanalyzed regarding: (1) demographic data, (2) age at tic onset, (3) tic severity according to the Shapiro Tourette Syndrome Severity Scale (STSS, including 5 items quantifying tic severity and interference, range, $0=$ no tics$6=$ very severe tics $^{19}$ ) at time of first consultation, (4) psychiatric comorbidities according to a semi-structured interview, ${ }^{1}(5)$ number of comorbidities (=comorbidity score, range $0-8)^{20},(6)$ treatment for GTS (ever), and (7) details about CBM treatment: type and number of different CBM, duration of treatment, dosage, acute and long-term beneficial effects on tics, comorbidities, and further symptoms, acute and long-term AEs, differences between different CBM, and-where appropriate-discontinuation of CBM treatment. As part of our routine clinical work, we ask patients about subjective improvements of different symptoms after taking CBM expressed as a percentage. Thus, the effects of CBM treatment on tics, comorbidities, and further symptoms were based on these subjective assessments by patients. Finally, we had a focus on information about formal, legal, and logistical aspects related to CBM such as financing, coverage of costs by health insurances, and existence of a license from the German Federal Institute for Drugs and Medical Devices (BfArM) for legal selfmedication with cannabis (that was required for legal use of cannabis in Germany before 03/2017).

\section{Part 2: online survey}

Patients were asked to complete an online survey (SoSci survey, between 03 and 09/2017) to receive detailed data about current treatment of GTS, including type, duration, and discontinuation of treatment, preferred CBM, effects of CBM on tics, comorbidities, premonitory urges, tic suppression, quality of life, AEs, route of intake, and formal, legal and logistical aspects related to CBM. The survey consisted of standard multiple- and single-choice questions as well as Likert scales (ranging from $1=$ not at all to $10=$ very strong) for assessing the degree of improvement of tics, premonitory urge, and quality of life. This survey was specially developed for this study and does not constitute a validated assessment instrument. All data were collected anonymously in accordance with applicable data protection laws. 


\section{Statistical analysis}

Data analysis was performed as a chart analysis by using Microsoft ${ }^{\circledR}$ Excel 16.6.1. Since some patients had used more than one CBM, for specific questions, it was possible to receive more than one answer from a single patient. Therefore, in both study parts, " $n$ " may refer either to the number of patients or to the number of "statements" (always indicated). Statements were individual answers on specific questions with a simple "yes" or "no" made by patients who used more than one CBM and were able to answer single questions regarding different substances. We performed statistical group comparisons among patients who received different CBM based on retrospective data of part 1 . The analyzed variables were: subjective degree of improvement (one-way analysis of variance [ANOVA]), number of comorbidities, and number of further symptoms (chi-square test). We carried out additional post hoc comparisons on the ANOVA as well as on the chi-square test. The contrasts for the ANOVA were computed via the Tukey post hoc test, and the contrasts for the chi-square test were computed via a method proposed by Beasley and Schumacker. ${ }^{21}$ This nonparametric contrast analysis is basically a contingency table analysis, which consists of the following steps: A chi-square test of independence is conducted between two variables of interest under the null hypothesis that the frequencies of all cells correspond to the product of the according row and column frequencies. In each cell, the actually observed frequency is compared with the expected frequency and a residual is being determined. In the further analysis, the residuals of all cells are transformed into chi-square scores and a $p$-value is determined. Finally, a Bonferroni correction is applied by taking into account the resulting number of comparisons.

\section{Results}

Part 1: retrospective analysis

We were able to identify 98 patients. Further clinical characteristics are summarized in Table 1 (due to incomplete data, numbers may vary).

Demographic characteristics. The majority of our patients had a school-leaving qualification $(96 \%)$ or even high level of education (64\%) and completed vocational training (49\%) or were currently in education/training (18\%). Moreover, $45 \%$ of subjects were employed at the moment of examination, $36 \%$ were unemployed, and $19 \%$ had retired.

Treatment with CBM. Before consultation, the patients received on average $5.8(\mathrm{SD}=4.2)$ different
Table 1. Part 1 (Retrospective Analysis): Clinical Characteristics

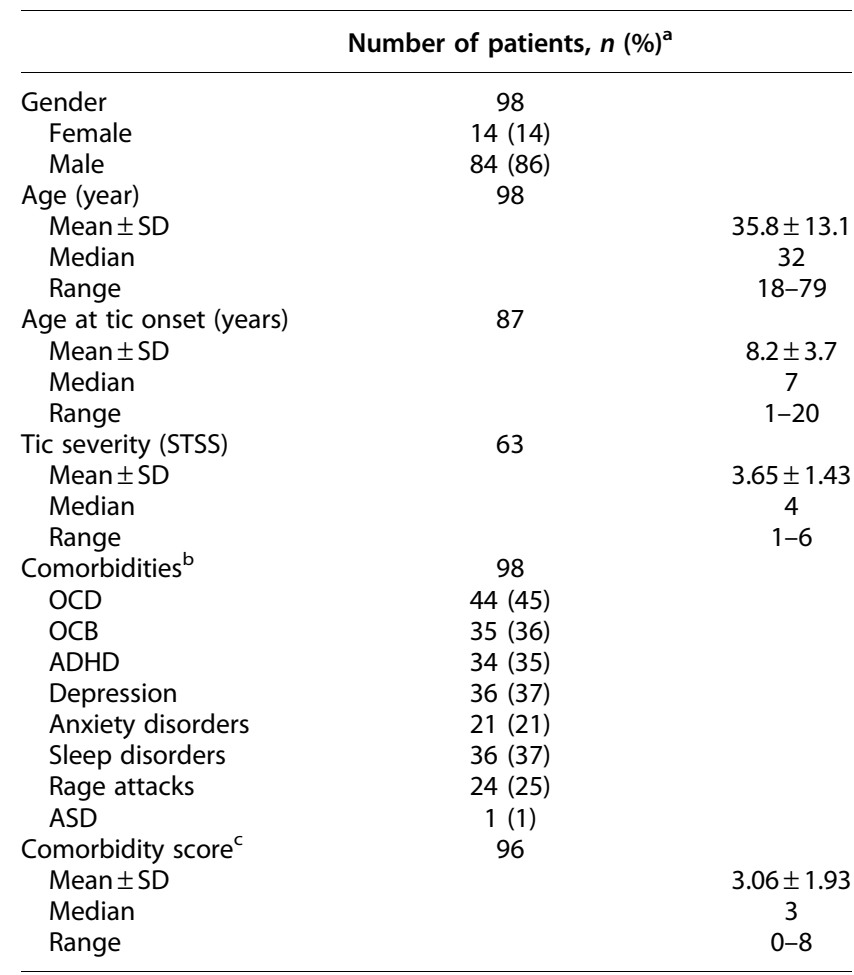

${ }^{a}$ Due to incomplete data, numbers may vary.

${ }^{b}$ Diagnoses according to semi-structured clinic interview.

'Number of comorbidities, range 0-8; STSS, Shapiro Tourette Syndrome Severity Scale, range 0-6.

$A D H D$, attention deficit/hyperactivity disorder; ASD, autism spectrum disorder; $\mathrm{OCB} / \mathrm{OCD}$, obsessive-compulsive behavior/disorder.

treatments for tics and comorbidities (range 0-20) and $90 \%$ of patients had received treatment with at least 1 antipsychotic. Twenty-five percent of patients had initiated treatment with CBM already before first consultation, another $20 \%$ specifically sought advice regarding CBM, and $26 \%$ of patients had received a license from BfArM for legal self-medication with cannabis. Sixty-nine patients (71\%) had used street cannabis for the treatment of GTS at some time point, 36 (37\%) nabiximols, 36 (37\%) dronabinol, and 21 (22\%) medicinal cannabis (from pharmacy) (multiple answers possible). Altogether, 29 patients (30\%) had used a combined treatment with CBM: Of these, 6 patients (21\%) used different CBM at the same time, and 22 (79\%) used CBM and at least one other substance (non-CBM). Half of the patients $(n=50 / 98,51 \%)$ had tried more than one CBM (mean $=1.65 \pm 0.76$, median $=2$, range $1-4, n=98$ ) for the treatment of GTS. Out of these, 38 patients (76\%) felt able to judge which CBM worked best for them: 25 patients (66\%) favored medicinal cannabis, 7 (18\%) dronabinol, 4 (11\%) 
nabiximols, and 2 (5\%) street cannabis (irrespective of whether patients tried the CBM or believed in the superiority of one $\mathrm{CBM}$ ).

Duration of treatment, dosage, and long-term effect. Mean duration of treatment with CBM was $62.1 \pm 73.9$ months (median $=41$, range $1-336, n=77$ ). Thirty-one patients $(33 \%)$ discontinued CBM (reasons not known). Preferred dosages varied markedly for all CBM. For cannabis, effective doses are hard to quantify due to different contents of cannabinoids and different inhalation patterns (Table 2). Altogether, in 62 out of 116 statements (53\%), the patients confirmed a consistent long-term effect of prescribed CBM (Table 2).

Effects on tics, comorbidities, further symptoms, and AEs. We were able to analyze up to 156 statements (from 98 patients) with respect to beneficial and side effects of CBM (since several patients had used more than one CBM), allowing to detect contrasting effects of different CBM even within one patient. Due to partly incomplete data, sizes of datasets differed. Altogether, $85 \%$ (133/156 statements) patients reported a tic improvement (on average of 60\%) (Table 3). With respect to psychiatric comorbidities, 55\% (47/86 statements) patients reported an improvement of one or more comorbidities, most often of $\mathrm{OCB} / \mathrm{OCD}, \mathrm{ADHD}$, and sleeping disorders (Table 3). In addition, 77\% (79/103 statements) patients reported further positive effects (Table 3). Finally, $45 \%$ (67/150 statements) patients reported no AEs and 55\% (83/150 statements) $\geq 1$ CBM-related AEs, most often (>10\%) dizziness, fatigue, and changed appetite (Table 4).
Effects depending on the type of CBM. For the subjective degree of improvement for tics, the overall one-way ANOVA revealed a significant subgroup difference $[F(3$, $152)=14.62, p<0.001]$. A contrast analysis via Tukey post hoc analysis revealed the following contrasts: Medicinal cannabis was significantly better than THC $(-35.00$, 95\% CI $[-56.23$ to -13.78$], p=0.0002)$ and nabiximols $(-37.00,95 \%$ CI $[-58.47$ to -15.53$], p=0.0001)$. In the same vein, street cannabis was significantly better than THC $(-29.00,95 \%$ CI $[-45.04$ to -12.96$], p<0.0001)$ and nabiximols $(-31.00,95 \%$ CI $[-47.36$ to -14.64$]$, $p<0.0001)$. There was no significant difference between street cannabis and medicinal cannabis $(p=0.85)$, nor between nabiximols and THC $(p=0.99)$. All comparisons withstood Bonferroni correction.

When improvement of tics was treated as a binary outcome, a significant subgroup difference was also detected $\left[\chi^{2}(3)=9.15, p<0.05\right]$. A contrast analysis showed that the improvement for street cannabis was significantly higher than expected under the assumption of independence [street cannabis: $\chi^{2}(1)=6.30$, $p=0.012]$. Improvement for THC was significantly lower than expected under the assumption of independence $\left[\chi^{2}(1)=4.75, p=0.029\right]$. Only street cannabis withstood Bonferroni correction. For the improvement of all comorbid conditions taken together, the overall Pearson chi-square test comparing all four medication groups showed significant difference $\left[\chi^{2}(3)=32.83\right.$, $p<0.001]$. A contrast analysis showed the improvement for street cannabis was significantly higher than expected under the assumption of independence [street cannabis: $\left.\chi^{2}(1)=11.83, p=0.0006\right]$. Improvement for

Table 2. Part 1 (Retrospective Analysis): Dosage and Long-Term Effect of Cannabis-Based Medicine (Only Prescribed Cannabis-Based Medicine Included)

\begin{tabular}{|c|c|c|c|c|}
\hline & $\begin{array}{c}\text { Medicinal cannabis } \\
{[\mathrm{g} / \text { day }]}\end{array}$ & $\begin{array}{c}\text { Dronabinol } \\
\text { [mg/day] }\end{array}$ & $\begin{array}{l}\text { Nabiximols } \\
\text { [puffs/day] }^{\mathrm{a}}\end{array}$ & $\sum \mathrm{CBM}^{\mathrm{b}}$ \\
\hline Total No. of statements & 21 & 36 & 36 & \\
\hline \multicolumn{5}{|l|}{ Dosage } \\
\hline Mean $\pm S D$ & $2.2 \pm 2.39$ & $43.2 \pm 68.32$ & $10.6 \pm 8.89$ & \\
\hline Median & 1.5 & 20 & 8 & \\
\hline Range & $0.2-10$ & $3-250$ & $3-40$ & \\
\hline \multicolumn{5}{|l|}{ Long-term effect } \\
\hline Constant effect (without dose adjustment), $n(\%)^{c}$ & $14 / 20(70)$ & $13 / 31(42)$ & $13 / 31(42)$ & $40 / 82(49)$ \\
\hline Decreasing effect (before dose adjustment), $n(\%)^{c}$ & $6 / 20(30)$ & $18 / 31(58)$ & $18 / 31(58)$ & $42 / 82(51)$ \\
\hline Constant effect (after dose adjustment), $n(\%)^{c}$ & $8 / 8(100)$ & 11/14 (79) & $3 / 12(25)$ & $22 / 34(65)$ \\
\hline Decreasing effect (despite dose adjustment), $n(\%)^{c}$ & $0 / 8(0)$ & $3 / 14(21)$ & $9 / 12(75)$ & $12 / 34(35)$ \\
\hline
\end{tabular}

${ }^{\mathrm{a} E}$ Each puff contains $2.7 \mathrm{mg}$ THC and $2.5 \mathrm{mg}$ CBD.

${ }^{b} \sum \mathrm{CBM}=$ sum of all CBM shown in the table.

'Due to incomplete data, numbers may vary. $n$ refers to number of statements about the use of a CBM, please note that this does not necessarily correspond to the number of patients, since patients could have used more than one CBM.

CBD, cannabidiol; CBM, cannabis-based medicine; THC, tetrahydrocannabinol. 
Table 3. Part 1 (Retrospective Analysis): Beneficial Effects of Cannabis-Based Medicine

\begin{tabular}{|c|c|c|c|c|c|}
\hline Improvement of & Medicinal cannabis & Dronabinol & Nabiximols & Street cannabis & $\sum \mathrm{CBM}^{\mathrm{a}}$ \\
\hline Tics, $n(\%)^{b}$ & $21 / 21(100)$ & $27 / 35(77)$ & $25 / 33(76)$ & $60 / 67(90)^{c}$ & $133 / 156(85)$ \\
\hline \multicolumn{6}{|l|}{ Degree of improvement [\%] ${ }^{d}$} \\
\hline Mean \pm SD & $79 \pm 13^{*}$ & $44 \pm 35^{*}$ & $42 \pm 32^{*}$ & $73 \pm 29^{*}$ & $60 \pm 27$ \\
\hline Median & 80 & 50 & 45 & 80 & 64 \\
\hline Range & $55-100$ & $0-90$ & $0-100$ & $0-100$ & $14-98$ \\
\hline$n(\%)^{b}$ & 15/21 (71) & 25/36 (69) & $18 / 36(50)$ & $38 / 49$ (78) & $96 / 142(68)$ \\
\hline Comorbidities, $n(\%)^{\mathrm{b}}$ & $10 / 13(77 \%)$ & $12 / 20(60 \%)$ & $3 / 20(15 \%)^{\mathrm{e}}$ & $22 / 33(67 \%)^{c}$ & $47 / 86(55 \%)$ \\
\hline OCD & $2 / 13(15 \%)$ & $5 / 12(42 \%)$ & $1 / 3(33 \%)$ & 9/33 (27\%) & $17(36 \%)$ \\
\hline $\mathrm{OCB}$ & $3 / 13(23 \%)$ & $6 / 12(50 \%)$ & $1 / 3(33 \%)$ & 11/33 (33\%) & $21(45 \%)$ \\
\hline $\mathrm{OCB} / \mathrm{OCD}$ & $5 / 13(38 \%)$ & $11 / 12(92 \%)$ & $2 / 3(67 \%)$ & 20/33 (61\%) & $38(81 \%)$ \\
\hline ADHD & $7 / 13(53 \%)$ & $3 / 12(25 \%)$ & $1 / 3(33 \%)$ & $5 / 33(15 \%)$ & $16(34 \%)$ \\
\hline Sleep disorders & $6 / 13(46 \%)$ & 10/12 (83\%) & $1 / 3(33 \%)$ & $18 / 33(55 \%)$ & $35(74 \%)$ \\
\hline Further symptoms, $n(\%)^{b}$ & $12 / 12(100 \%)$ & $16 / 22(72 \%)$ & $11 / 24(46 \%)^{\mathrm{e}}$ & $40 / 45(89 \%)$ & $79 / 103(77 \%)$ \\
\hline Improved restlessness & $8 / 12(67 \%)$ & $10 / 16(63 \%)$ & 7/11 (64\%) & $28 / 40(70 \%)$ & $53(67 \%)$ \\
\hline Less quick-tempered behavior & $8 / 12(67 \%)$ & $9 / 16(56 \%)$ & $4 / 11(36 \%)$ & $9 / 40(23 \%)^{\mathrm{e}}$ & $30(38 \%)$ \\
\hline Improved mood & 4/12 (33\%) & $3 / 16(19 \%)$ & 4/11 (36\%) & $12 / 40(30 \%)$ & $23(29 \%)$ \\
\hline General relaxation & 4/12 (33\%) & $2 / 16(13 \%)$ & $1 / 11(9 \%)$ & $12 / 40(30 \%)$ & $19(24 \%)$ \\
\hline
\end{tabular}

Statistical comparisons were conducted via chi-square test and one-way ANOVA. Contrasts were corrected for multiple comparisons via Bonferroni correction.

${ }^{*} p<0.001$.

a $\sum \mathrm{CBM}=$ sum of all CBM shown in the table.

$\mathrm{b}_{n}$ refers to number of statements (not number of patients) describing a symptom improvement, since patients could have used more than one CBM.

'Significantly higher than expected under the assumption of independence.

'Degree of improvement based on patients' subjective assessments expressed as a percentage.

'Significantly lower than expected under the assumption of independence.

ANOVA, analysis of variance.

Table 4. Part 1 (Retrospective Analysis): Adverse Effects of Cannabis-Based Medicine

\begin{tabular}{|c|c|c|c|c|c|}
\hline & $\begin{array}{c}\text { Medicinal } \\
\text { cannabis, } \boldsymbol{n}(\%)\end{array}$ & $\begin{array}{c}\text { Dronabinol, } \\
n(\%)\end{array}$ & $\begin{array}{c}\text { Nabiximols, } \\
n(\%)\end{array}$ & $\begin{array}{c}\text { Street cannabis, } \\
n(\%)\end{array}$ & $\sum_{n(\%)}$ CBM, \\
\hline$N^{\mathrm{a}}$ & 19 & 36 & 31 & 64 & 150 \\
\hline No AEs & $11(58)$ & $13(36)$ & $6(19)$ & $37(58)$ & $67(45)$ \\
\hline AEs & $8(42)$ & $23(64)$ & $25(81)^{\mathrm{b}}$ & $27(42)^{c}$ & $83(55)$ \\
\hline \multicolumn{6}{|l|}{ Type of AE } \\
\hline Neurological disorders & $9(47)$ & $33(92)^{b}$ & $31(100)^{b}$ & $23(36)^{c}$ & $96(64)$ \\
\hline Dizziness & $3(16)$ & $12(33)$ & $10(32)$ & $6(9)$ & $31(21)$ \\
\hline Fatigue & $1(5)$ & 9 (25) & 11 (35) & $5(8)$ & $26(17)$ \\
\hline Weakness of memory or concentration & $1(5)$ & 4 (11) & $0(0)$ & $3(5)$ & $8(5)$ \\
\hline Agitation & $1(5)$ & $2(6)$ & 1 (3) & $2(3)$ & $6(4)$ \\
\hline Confusion/disorientation & $0(0)$ & $2(6)$ & $3(10)$ & $1(2)$ & $6(4)$ \\
\hline Discomfort or sensation of drunkenness & $1(5)$ & $0(0)$ & $3(10)$ & $2(3)$ & $6(4)$ \\
\hline Auditory and visual hallucinations & $0(0)$ & $1(3)$ & $2(6)$ & $1(2)$ & $4(3)$ \\
\hline Depression & $2(11)$ & $0(0)$ & $1(3)$ & $1(2)$ & $4(3)$ \\
\hline Feeling of weakness or general malaise & $0(0)$ & $2(6)$ & $0(0)$ & $1(2)$ & $3(2)$ \\
\hline Delusions & $0(0)$ & $0(0)$ & $0(0)$ & $1(2)$ & $1(1)$ \\
\hline Dysarthria & $0(0)$ & $1(3)$ & $0(0)$ & $0(0)$ & $1(1)$ \\
\hline Eye disorders & $0(0)$ & $2(6)$ & $0(0)$ & $0(0)$ & $2(1)$ \\
\hline Vision disturbances & $0(0)$ & $2(6)$ & $0(0)$ & $0(0)$ & $2(1)$ \\
\hline Gastrointestinal disorders & $3(16)$ & $14(39)$ & $22(71)^{b}$ & $7(11)^{c}$ & $56(37)$ \\
\hline Increased or reduced appetite & $2(11)$ & $6(17)$ & 7 (23) & $5(8)$ & 20 (13) \\
\hline Constipation or diarrhea & $0(0)$ & $5(14)$ & $2(6)$ & $0(0)$ & 17 (11) \\
\hline Altered taste or dry mouth & $1(5)$ & $2(6)$ & 9 (29) & $0(0)$ & $12(8)$ \\
\hline Oral paresthesia & $0(0)$ & $0(0)$ & $2(6)$ & $1(2)$ & $3(2)$ \\
\hline Nausea and vomiting & $0(0)$ & $0(0)$ & $2(6)$ & $0(0)$ & $2(1)$ \\
\hline Abdominal pain & $0(0)$ & $1(3)$ & $0(0)$ & $0(0)$ & $1(1)$ \\
\hline Throat dryness or coughing & $0(0)$ & $0(0)$ & $0(0)$ & $1(2)$ & $1(1)$ \\
\hline Cardiac disorders & $0(0)$ & $0(0)$ & $1(3)$ & $0(0)$ & $1(1)$ \\
\hline Changes in heart rate or blood pressure & $0(0)$ & $0(0)$ & $1(3)$ & $0(0)$ & $1(1)$ \\
\hline
\end{tabular}

Adverse effects reported in $>10 \%$ are shown in bold.

${ }^{a}$ Multiple answers possible, resulting in $n$ statements.

${ }^{\mathrm{b}}$ Significantly higher than expected under the assumption of independence.

'Significantly lower than expected under the assumption of independence. Statistical comparisons were conducted via chi-square test and oneway ANOVA. Contrasts were corrected for multiple comparisons via Bonferroni correction.

AEs, adverse events. 
nabiximols was significantly lower than expected under the assumption of independence $\left[\chi^{2}(1)=23.04\right.$, $p=0.0001]$. Both contrasts withstood corrections for multiple comparisons.

With respect to the improvement of specific comorbidities, the overall Pearson chi-square test comparing all four medication groups did not show a significant difference [OCD: $\chi^{2}(3)=2.195, p=0.53$, OCB: $\chi^{2}(3)=2.050$, $p=0.56$, OCB/OCD: $\chi^{2}(3)=7.62, p=0.055$, ADHD: $\chi^{2}(3)=7.305, p=0.06$, sleeping disorders: $\chi^{2}(3)=4.793$, $p=0.18]$. Notably, the OCB/OCD and ADHD missed significance by a small margin. There is a trend for dronabinol and street cannabis to have similar effects on $\mathrm{OCB} / \mathrm{OCD}$, whereas there is a trend for medicinal cannabis to be more effective on ADHD (Table 3 and Fig. 1).

For the improvement of all further symptoms taken together, the overall Pearson chi-square test comparing all four medication groups showed significant difference $\left[\chi^{2}(3)=18.90, p<0.001\right]$. A contrast analysis showed that street and medical cannabis had a significantly better effect [street cannabis: $\chi^{2}(1)=5.48, p=0.019$; medicinal cannabis: $\chi^{2}(1)=4.28, p=0.039$ ] than the other CBM. The effect of nabiximols was significantly below the average effect $\left[\chi^{2}(1)=15.52, p=0.0001\right]$. The effect of THC was not significant $\left[\chi^{2}(1)=0.16, p=0.69\right]$. Only the effect of nabiximols withstood corrections for multiple comparisons.

For comparison of $\mathrm{AE}$ among the four medication groups, the overall Pearson chi-square test showed a significant difference $\left[\chi^{2}(3)=14.92, p<0.05\right]$. A contrast analysis showed that on nabiximols the rate of reported $\mathrm{AE}$ was significantly higher than expected under the assumption of independence $\left[\chi^{2}(1)=10.11\right.$, $p=0.0015]$, whereas on street cannabis it was significantly lower than expected under the assumption of independence $\left[\chi^{2}(1)=7.78, p=0.0053\right]$. Both contrasts withstood corrections for multiple comparisons. For intergroup differences of different AEs see Table 4.

\section{Part 2: online survey}

Forty out of 98 patients (41\%) participated in the second part. Reasons for noninclusion were: lost for follow-up ( $n=24,25 \%)$, no interest $(n=15,15 \%)$, noncompliance $(n=16,16 \%)$, and other reasons $(n=3,3 \%)$.

Current medication. Thirty-five patients (87\%) indicated to be currently receiving medical treatment for GTS; 23 out of 40 patients (57\%) received treatment with $\geq 1$ of the following CBM (multiple answers possible): medicinal cannabis: $n=9$ (39\%), nabiximols: $n=6(26 \%)$, dronabinol: $n=4(17 \%)$, and street cannabis: $n=8(35 \%)$. Of these, 17 patients $(74 \%)$ reported a treatment duration $>1$ year. Eleven patients (28\%) received combined treatment for tics with CBM and antipsychotics.

Effects of different CBM. We received 27 statements from 23 patients (multiple answers possible) regarding specific effects of CBM: whereas almost all patients-independently of the type of $\mathrm{CBM}$-reported an

Table 5. Part 2 (Online Survey): Improvement of Tics, Premonitory Urges, Tic Suppression, Quality of Life, and Number of Patients Reporting At Least One Adverse Event Depending on the Type of Cannabis-Based Medicine

\begin{tabular}{|c|c|c|c|c|c|}
\hline & Medicinal cannabis & Dronabinol & Nabiximols & Street cannabis & $\sum \mathrm{CBM}^{\mathrm{a}}$ \\
\hline$N^{b}$ & 9 & 4 & 6 & 8 & 27 \\
\hline Tics, $n(\%)$ & $9(100)$ & $4(100)$ & $5(83)$ & $8(100)$ & $26(96)$ \\
\hline \multicolumn{6}{|c|}{ Degree of improvement ${ }^{c}$} \\
\hline Mean $\pm S D$ & $7.89 \pm 0.6$ & $6.75 \pm 1.89$ & $5.2 \pm 1.79$ & $7.44 \pm 1.94$ & $6.82 \pm 1.56$ \\
\hline Median & 8 & 7.5 & 4 & 8 & 7 \\
\hline Range & $7-9$ & $4-8$ & $4-8$ & $4-10$ & $4-10$ \\
\hline Premonitory urge, $n$ (\%) & 7 (78) & $1(25)$ & $2(33)$ & $6(75)$ & $16(59)$ \\
\hline \multicolumn{6}{|l|}{ Degree of improvement ${ }^{c}$} \\
\hline Mean $\pm S D$ & $6.86 \pm 1.77$ & $1.33 \pm 0.58$ & $4 \pm 1.41$ & $6.83 \pm 1.47$ & $4.76 \pm 5.23$ \\
\hline Median & 7 & 1 & 4 & 7 & 5 \\
\hline Range & $4-9$ & $1-2$ & $3-5$ & $4-8$ & $1-9$ \\
\hline Tic suppression, $n(\%)$ & $9(100)$ & $3(75)$ & $4(67)$ & $8(100)$ & $20(74)$ \\
\hline Quality of life, $n(\%)$ & $9(100)$ & $4(100)$ & $4(67)$ & $8(100)$ & $25(93)$ \\
\hline \multicolumn{6}{|l|}{ Degree of improvement ${ }^{c}$} \\
\hline Mean $\pm S D$ & $9 \pm 0.87$ & $8.25 \pm 0.5$ & $8.75 \pm 0.96$ & $7.63 \pm 1.85$ & $8.41 \pm 1$ \\
\hline Median & 9 & 8 & 8.5 & 8 & 8.5 \\
\hline Range & $8-10$ & $8-9$ & $8-10$ & $5-10$ & $5-10$ \\
\hline $\mathrm{AEs}, n(\%)$ & $9(33)$ & $4(25)$ & $6(50)$ & $8(13)$ & $27(30)$ \\
\hline
\end{tabular}

${ }^{a} \mathrm{C} C B M=$ sum of all CBM shown in the table.

${ }^{b} n$ refers to number of statements (not number of patients), since patients could have used more than one CBM. Given are numbers of statements describing a symptom improvement.

${ }^{c}$ Degree of improvement obtained from a self-rated Likert scale ${ }^{1-10}: 1=$ not at all, $10=$ very strong. 


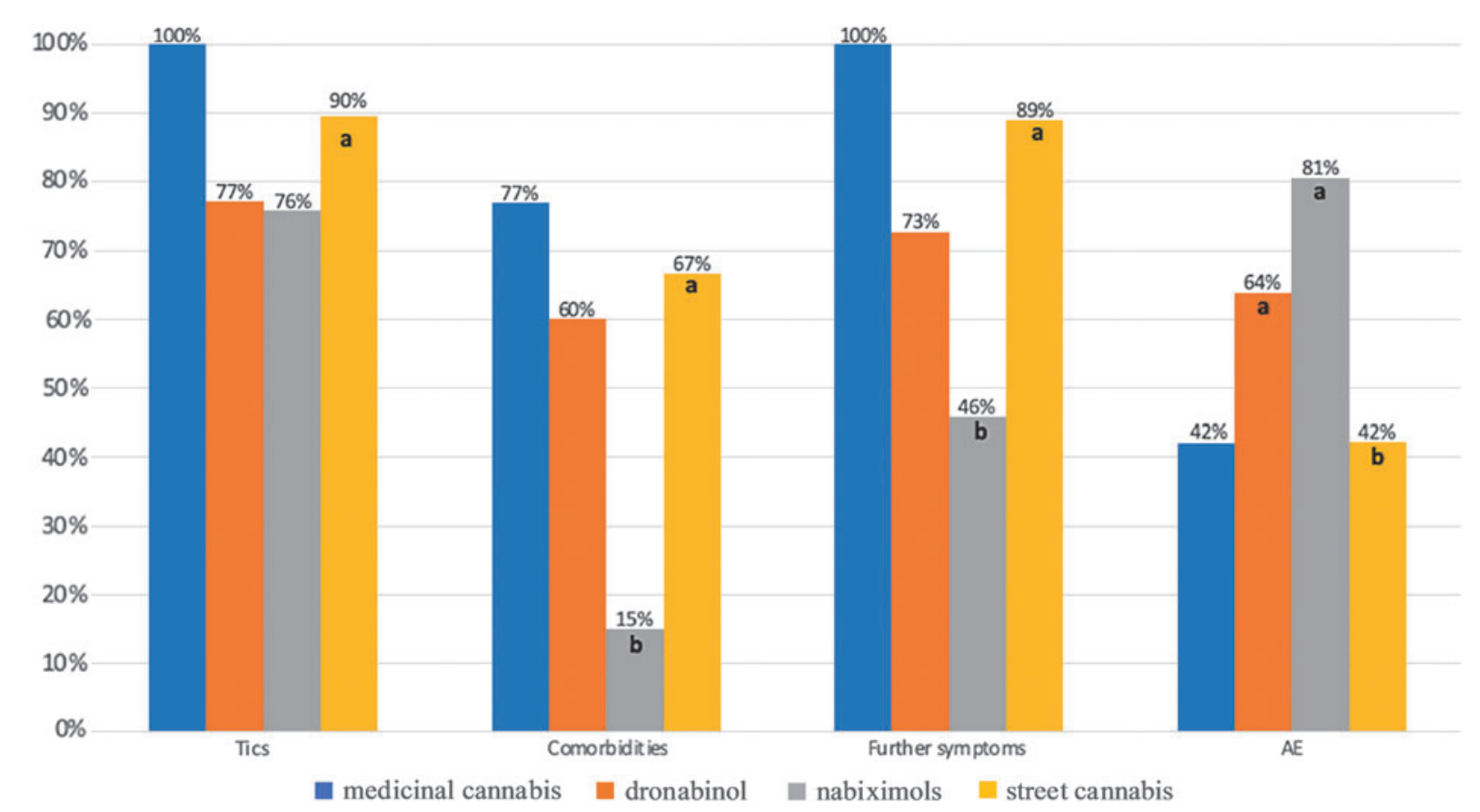

FIG. 1. Part 1 (retrospective analysis): percentages of patients reporting improvement of tics, comorbidities, and further symptoms and confirming AEs depending on the type of CBM. Percentages refer to varying number of patients' statements. Data based on small sample sizes ranging from 86 to 156 statements obtained from 12 to 67 patients. Absolute values are given in Table 5. ${ }^{\mathrm{a}}$ Significantly higher than expected under the assumption of independence, ${ }^{\mathrm{b}}$ significantly lower than expected under the assumption of independence. Statistical comparisons were conducted via chi-square test and one-way ANOVA. Contrasts were corrected for multiple comparisons via Bonferroni correction. AEs, adverse events; ANOVA, analysis of variance; CBM, cannabis-based medicine.

improvement of tics (96\%) and quality of life (93\%), (street and medicinal) cannabis seem to have stronger effects on premonitory urges and tic suppression (Table 5). AEs were most often reported after nabiximols, followed by medicinal cannabis, dronabinol, and street cannabis (Table 5). Based on 57 statements from 40 patients (multiple answers possible), patients rated "medicinal cannabis" as "best prescribed CBM" (data not shown). In terms of effects of different cannabis strains, 9 patients provided a total of 24 statements (on 10 different cannabis strains containing 1\% to $22 \%$ THC). Based on these, THC-rich strains with a THC content $>15 \%$ were prescribed by far more often ( $n=17 / 24$ statements) compared with strains with low to medium THC content. The most frequently used strain had a THC content of $22 \%(n=17 / 24$ statements).

Route of intake. Due to small sample sizes and incomplete data, no final conclusion can be drawn with respect to the preferred route of intake, but patients used the whole spectrum from oral intake to smoking (no data shown).

Discontinuation of CBM. Between the first and second study part, 17 out of 40 patients (43\%) had stopped treatment with the following CBM (multiple answers possible): street cannabis: $n=13$ (76\%), nabiximols: $n=9$ (53\%), medicinal cannabis: $n=8$ (47\%), and dronabinol: $n=6$ (35\%) (we did not collect information on the reasons for discontinuation of cannabis).

Practical and financial aspects. Nine out of 40 patients (23\%) had received a license from BfArM (before 03/2017) and 8 out of 9 (89\%) had utilized it for legal self-medication with medicinal cannabis from the pharmacy (on their own expenses). Overall, 26 patients (65\%) stated that they had to cover costs for CBM by themselves at some time. In only $40 \%$ of the patients $(n=16)$, health insurances agreed to cover the costs for CBM. More than half of the patients $(n=23,57 \%)$ 
reported about a substantial financial burden associated with CBM treatment significantly influencing treatment.

\section{Discussion}

To the best of our knowledge, this is not only the largest dataset available but also the first study directly comparing effects of different CBM in patients with GTS. The major findings of this study are: (1) CBM is effective in the treatment of tics and comorbidities in a quite large percentage of patients, resulting in a relevant improvement of quality of life, (2) CBM improves premonitory urges and tic suppressibility, (3) cannabis seems to be more effective and better tolerated compared with nabiximols and dronabinol, (4) patients prefer cannabis with medium to high THC content, (5) the effects of CBM appear to persist in the long term, since patients continued using them for years, and (6) AEs in patients with GTS do not differ from those in patients with other diseases such as multiple sclerosis $^{22}$ and chronic neuropathic pain. ${ }^{23}$

Treating physicians need to recognize that a substantial number of patients with GTS use CBM to improve tics, comorbidities, and overall quality of life. However, until today, the database is weak and there are only results available from single case reports, ${ }^{9,10,12-18}$ three small retrospective surveys (including 17, 19, and 24 patients, respectively) using (street or medicinal) cannabis, ${ }^{5,6,11}$ and two small RCTs using dronabinol (including 12 and 24 patients, respectively). ${ }^{7,8}$ Since over the past years an increasing number of different CBM became available, we were interested in investigating for the first time (beneficial and adverse) effects of different types of CBM. Due to costs and availability in Germany, the only CBM used in our patients' group were (street and medicinal) cannabis, dronabinol, and nabiximols.

In both study parts, the vast majority of patients reported an improvement of their tics after CBM. Similar to available studies, ${ }^{5-18}$ patients assessed that, on average, tics improved by $60 \%$ ("much improved"). Although all CBM were reported to be effective, cannabis seems to be the most effective CBM. Most interestingly, patients reported not only an improvement of tics but also an improvement of premonitory urges before the occurrence of the tics and an improved ability of voluntary tic suppression. Since it is believed that premonitory urges are a core symptom of the disease, ${ }^{24,25}$ one might speculate that CBM improves tics right from the beginning. So far, there is only very limited evidence that other pharmacological treatments may also improve premonitory urges. ${ }^{26}$ From our results, however, it cannot be excluded that tics and urges improved-at least in part-only secondary due to reduced anxiety, stress, or tension caused by CBM.

In agreement with earlier studies, ${ }^{5,6,8,11-13,16,18}$ more than half of the patients reported, in addition, an improvement of behavioral symptoms, mainly comorbid $\mathrm{OCB} / \mathrm{OCD}, \mathrm{ADHD}$, and sleeping disorders and more than three-quarter of patients felt that CBM had additional positive effects such as improved restlessness and general mood, less quick-tempered behavior, and general relaxation. Altogether, more than $90 \%$ of patients reported an overall improvement of quality of life.

Comparable to other studies using CBM, ${ }^{27,28}$ about half of the patients reported AEs, most often dizziness, fatigue, and changed appetite. Noteworthy, most patients felt that (medicinal and street) cannabis causes less AEs compared with dronabinol and nabiximols. Since several patients used combined treatment for tics with either two different CBM or a CBM plus an antipsychotic (most often aripiprazole), it seems that in these cases combined treatment is more effective, but not limited by more frequent and severe AEs. The fact that yet about one third of patients stopped treatment with CBM seems to be best explained by financial burden, limited access to legal CBM, and avoidance of illegal use of street cannabis.

Dosages of all CBM significantly differed interindividually and ranged from very low to high dosages. On average, dosages were comparable to dosages used in other indications ${ }^{26,28,29}$ : cannabis: $1.5-2 \mathrm{~g} /$ day, dronabinol: $20-40 \mathrm{mg} /$ day, and nabiximols: $8-12$ puffs/day. Since several patients reported use of CBM for more than 1 year (up to 28 years), from our data it is suggested that-after an initial dose adjustment-beneficial effects of CBM do not decrease after long-term treatment due to habituation.

With respect to cannabis preparations, patients seem to prefer strains with higher (>15\%) THC content. Although this impression is based on a small number of patients, it is completely in line with our clinical experience: Most patients compare effects of different strains and usually decide for those with higher THC content, suggesting that THC-rich strains are more effective in TS. This is also in line with current literature, since there is no single case report available suggesting the efficacy of pure CBD in TS. As suggested earlier, ${ }^{30}$ we found no evidence that efficacy depends on cannabis species (sativa vs. indica).

Comparable to other surveys, ${ }^{31}$ patients reported that cannabis is more effective and better tolerated than 
nabiximols and dronabinol. A possible explanation might be the "entourage effect," suggesting that nonpsychoactive compounds of cannabis-such as terpenesenhance the action of the endogenous cannabinoid anandamide (through increased affinity to receptors or decreased enzymatic degradation) and that CBD mitigates some of the AEs of THC. This may result in improved tolerability, higher dosages of CBM, and, therefore, improved overall efficacy. ${ }^{17,32,33}$ Alternatively, patients' judgment may be influenced by the route of intake, since cannabis is primarily used via inhalation, whereas the other CBM are taken orally. The fact that nabiximols was rated as the worst CBM might be related to typical AEs due to intake via mouth spray (such as burning, pain, or ulcers in the mouth ${ }^{34}$ ).

Compared with other large samples ${ }^{1,20,35}$ we believe that our sample is representative for an adult clinic population regarding age, age at tic onset, and comorbidities. However, our sample comprised more male subjects (sex ratio $=6: 1$ ), patients with more severe tics (mean tic severity according to STSS: 3.65 vs. $2.79^{1}$ and $3.26^{35}$ respectively, in other samples), and subjects with a larger number of pretreatments (on average six different treatments before CBM). Of note, education level and employment rate in our sample were comparable to other large adult GTS samples, ${ }^{36-38}$ suggesting that use of CBM (including street cannabis) is not associated with lower education qualifications and higher levels of unemployment.

The following limitations of our study have to be addressed: (1) This is an open uncontrolled study based on a retrospective chart analysis (part 1) and an online survey; due to the study design, there was a large number of missing data that might have influenced the data; (2) although this is the largest sample investigating efficacy and safety of CBM in patients with GTS, the analyses conducted in this study still rely only on a small number of participants. Therefore, our results can only be viewed as interesting trends; (3) improvements based on patients' subjective assessments were expressed as a percentage and reported as average scores. Thus, results should be considered as preliminary and require replication in controlled studies using validated measurements; (4) diagnoses of comorbidities were made only based on a semi-structured clinical interview; for tic assessment the STSS was used instead of the gold standard for tic assessment (Yale Global Tic Severity Scale ${ }^{39}$ ); however, since all patients have been investigated by one of the authors, who is experienced in GTS (K.M.V.), we believe that data are reliable; (5) although our sample is compa- rable to samples in other studies including adults with GTS, ${ }^{1,20,34-37}$ we cannot entirely exclude that our sample is biased and not representative, since our sample comprised more male and more severely affected patients and a large number of patients, who had used street cannabis earlier; (6) it cannot be excluded that our results are influenced by effects of other pharmacological and nonpharmacological therapies; (7) presumably, our data are influenced by several formal aspects, including the legal situation and financial aspects.

\section{Conclusions}

Our results are in line with a number of other studies suggesting that CBM are effective and well tolerated at least in a subgroup of adults with GTS. From our data, it is suggested that CBM might be a treatment option even in those patients who are unsatisfied with established treatment strategies, Further, according to our data, CBM seems to be the first group of substances that has a long-lasting effect not only on tics, premonitory urges, and tic suppressibility but also on a wide range of psychiatric comorbidities, further symptoms, and patients' overall quality of life. In our sample, patients favored THC-rich cannabis over dronabinol and nabiximols. Because of several limitations of our study due to the open, uncontrolled design and the retrospective data analysis, controlled studies are needed to corroborate the preliminary findings.

\section{Acknowledgment}

The authors thank all patients for participating in this study.

\section{Author Disclosure Statement}

K.R.M.-V. has received consultant's honoraria from Abide Therapeutics, Fundacion Canna, and Therapix Biosiences, and speaker's fees from Tilray, and is a consultant for Zynerba Pharmaceuticals, has received financial or material research support from the EU (FP7HEALTH-2011 No. 278367, FP7-PEOPLE-2012-ITN No. 316978), the German Research Foundation (DFG: GZ MU 1527/3-1), the German Ministry of Education and Research (BMBF: 01KG1421), the National Institute of Mental Health (NIMH), Tourette Gesellschaft Deutschland e.V., Else-Kroner-Fresenius-Stiftung, and GW, Almirall, Abide Therapeutics, and Therapix Biosiences, consultant's honoraria from Abide Therapeutics and Therapix Biosiences, and royalties from Medizinisch Wissenschaftliche Verlagsgesellschaft Berlin. All other authors have no competing financial interests to declare. 


\section{Funding Information}

No funding was received for this article.

\section{References}

1. Sambrani T, Jakubovski E, Müller Vahl K. New insights into clinical characteristics of Gilles de la Tourette Syndrome: findings in 1032 patients from a single German Center. Front Neurosci. 2016;10:415-415.

2. Robertson M, Eapen V, Singer H, et al. Gilles de la Tourette syndrome. Nat Rev Dis Primers. 2017;3:16097-16097.

3. Roessner V, Plessen K, Rothenberger A, et al. European clinical guidelines for Tourette syndrome and other tic disorders. Part II: pharmacological treatment. Eur Child Adolesc Psychiatry. 2011;20:173-196.

4. Carulla-Roig $M$, Isomura $K$, Pérez-Vigil $A$, et al. Pharmacoepidemiology of Tourette and chronic tic disorders in Sweden. 2005-2013. J Child Adolesc Psychopharmacol. 2019. [Epub ahead of print]; http://doi.org/10.1089/ cap.2017.0169

5. Muller Vahl K, Kolbe H, Schneider U, et al. Cannabinoids: possible role in patho-physiology and therapy of Gilles de la Tourette syndrome. Acta Psychiatr Scand. 1998;98:502-506.

6. Abi-Jaoude $E$, Chen L, Cheung P, et al. Preliminary evidence on cannabis effectiveness and tolerability for adults with Tourette Syndrome. J Neuropsychiatry Clin Neurosci. 2017. appi.neuropsych; Available at: http:// psychiatryonline.org/doi/10.1176/appi.neuropsych.16110310 Accessed August 20, 2018.

7. Müller Vahl KR, Schneider U, Koblenz A, et al. Treatment of Tourette's syndrome with Delta 9-tetrahydrocannabinol (THC): a randomized crossover trial. Pharmacopsychiatry. 2002;35:57-61.

8. Müller Vahl K, Schneider U, Prevedel H, et al. Delta 9tetrahydrocannabinol (THC) is effective in the treatment of tics in Tourette syndrome: a 6-week randomized trial. J Clin Psychiatry. 2003;64: 459-465.

9. Trainor D, Evans L, Bird R. Severe motor and vocal tics controlled with Sativex ${ }^{\circledR}$. Australas Psychiatry. 2016;24:541-544.

10. Kanaan A, Jakubovski E, Müller Vahl K. Significant tic reduction in an otherwise treatment-resistant patient with Gilles de la Tourette Syndrome following treatment with nabiximols. Brain Sci. 2017;7. DOI: 10.3390/brainsci7050047.

11. Thaler A, Arad S, Schleider LB, et al. Single center experience with medical cannabis in Gilles de la Tourette syndrome. Parkinsonism Relat Disord. 2019;61:211-213.

12. Jakubovski E, Müller Vahl K. Speechlessness in Gilles de la Tourette Syndrome: cannabis-based medicines improve severe vocal blocking tics in two patients. Int J Mol Sci. 2017;18. DOI: 10.3390/ijms18081739.

13. Brunnauer A, Segmiller F, Volkamer T, et al. Cannabinoids improve driving ability in a Tourette's patient. Psychiatry Res. 2011;190:382.

14. Hasan A, Rothenberger A, Münchau A, et al. Oral delta 9tetrahydrocannabinol improved refractory Gilles de la Tourette syndrome in an adolescent by increasing intracortical inhibition: a case report. J Clin Psychopharmacol. 2010;30:190-192.

15. Hemming $M$, Yellowlees PM. Effective treatment of Tourette's syndrome with marijuana. J Psychopharmacol (Oxford). 1993;7:389-391.

16. Müller Vahl KR, Schneider U, Kolbe $H$, et al. Treatment of Tourette's syndrome with delta-9-tetrahydrocannabinol. Am J Psychiatry. 1999;156:495.

17. Pichler E, Kawohl W, Seifritz E, et al. Pure delta-9-tetrahydrocannabinol and its combination with cannabidiol in treatment-resistant Tourette syndrome: a case report. Int J Psychiatry Med. 2019;54:150-156.

18. Sandyk R. Self-mutilation in Tourette's syndrome. J Child Neurol. 1988;3: 147-148.

19. Shapiro A, Shapiro E, Young J, et al. Measurement in tic disorders. In: Shapiro A, Shapiro E, Young J, Feinberg T, eds. Gilles de la Tourette syndrome. 2nd ed. New York: Raven Press, 1988, pp. 451-480.

20. Freeman RD, Fast DK, Burd L, et al. An international perspective on Tourette syndrome: selected findings from 3,500 individuals in 22 countries. Dev Med Child Neurol. 2000;42:436-447.

21. Beasley TM, Schumacker RE. Multiple regression approach to analyzing contingencys: post hoc and planned comparison preocedures. J Exp Educ. 1995;64:79-93.

22. Novotna A, Mares J, Ratcliffe S. A randomized, double-blind, placebocontrolled, parallel-group, enriched-design study of nabiximols* (Sati- vex $\left.{ }^{\circledR}\right)$, as add-on therapy, in subjects with refractory spasticity caused by multiple sclerosis. Eur J Neurol. 2011;18:1122-1131.

23. Petzke F, Enax-Krumova EK, Häuser W. Efficacy, tolerability and safety of cannabinoids for chronic neuropathic pain: a systematic review of randomized controlled studies. Schmerz. 2016;30:62-88.

24. Leckman JF, Walker DE, Cohen DJ. Premonitory urges in Tourette's syndrome. Am J Psychiatry. 1993;150:98-102.

25. Cox J, Seri S, Cavanna A. Sensory aspects of Tourette syndrome. Neurosci Biobehav Rev. 2018;88:170-176.

26. Leckman JF, Bloch MH, Sukhodolsky DG, et al. Phenomenology of tics and sensory urges: the self under siege. In: Martino D, Leckmann JF, eds. Tourette syndrome. New York: Oxford University Press Inc., 2013. pp. 3-25.

27. Whiting P, Wolff R, Deshpande $S$, et al. Cannabinoids for medical use: a systematic review and meta-analysis. JAMA. 2015;313:2456-2473.

28. Wang T, Collet J, Shapiro S, et al. Adverse effects of medical cannabinoids: a systematic review. CMAJ. 2008;178:1669-1678.

29. Lim K, See Y, Lee J. A systematic review of the effectiveness of medical cannabis for psychiatric, movement and neurodegenerative disorders. Clin Psychopharmacol Neurosci. 2017;15:301-312.

30. Piomelli D, Russo E. The Cannabis sativa versus Cannabis indica debate: an interview with Ethan Russo, MD. Cannabis Cannabinoid Res 2016;1:44-46. DOI: 10.1089/can.2015.29003.ebr. eCollection.

31. Hazekamp A, Ware M, Muller Vahl K, et al. The medicinal use of cannabis and cannabinoids - an international cross-sectional survey on administration forms. J Psychoactive Drugs. 2013;45:199-210.

32. Mechoulam R, Fride E, Di Marzo V. Endocannabinoids. Eur J Pharmacol. 1998;359:1-18.

33. Niesink RJM, van Laar MW. Does cannabidiol protect against adverse psychological effects of THC?. Front Psychiatry. 2013;4:130.

34. Cannabinoid buccal spray for chronic non-cancer or neuropathic pain: a review of clinical effectiveness, safety, and guidlines [Internet]. Ottawa (ON): Canadian Agency for Drugs and Technologies in Health, 2016. Available at: www.ncbi.nlm.nih.gov/books/NBK395789 Accessed September 28, 2018.

35. Dodel I, Reese J, Müller N, et al. Cost of illness in patients with Gilles de la Tourette's syndrome. J Neurol. 2010;257:1055-1061.

36. Conelea C, Woods D, Zinner S, et al. The impact of Tourette Syndrome in adults: results from the Tourette Syndrome impact survey. Community Ment Health J. 2013;49:110-120.

37. Yang J, Hirsch L, Martino D, et al. The prevalence of diagnosed tourette syndrome in Canada: a national population-based study. Mov Disord. 2016;31:1658-1663.

38. Elstner K, Selai CE, Trimble MR, et al. Quality of Life (QOL) of patients with Gilles de la Tourette's syndrome. Acta Psychiatr Scand. 2001;103:52-59.

39. Leckman JF, Riddle MA, Hardin MT, et al. The Yale Global Tic Severity Scale: initial testing of a clinician-rated scale of tic severity. J Am Acad Child Adolesc Psychiatry. 1989;28:566-573.

Cite this article as: Milosev LM, Psathakis N, Szejko N, Jakubovski E, Müller-Vahl KR (2019) Treatment of Gilles de la Tourette syndrome with cannabis-based medicine: results from a retrospective analysis and online survey, Cannabis and Cannabinoid Research 4:4, 265-274, DOI: $10.1089 /$ can.2018.0050.

\section{Abbreviations used}

$\mathrm{ADHD}=$ attention deficit/hyperactivity disorder ANOVA $=$ analysis of variance

BfArM $=$ German Federal Institute for Drugs and Medical Devices

$\mathrm{CBD}=$ cannabidiol

$\mathrm{CBM}=$ cannabis-based medicine

GTS $=$ Gilles de la Tourette syndrome

$\mathrm{OCB}=$ obsessive-compulsive behavior

$\mathrm{OCD}=$ obsessive-compulsive disorder

$\mathrm{RCT}=$ randomized controlled trial

STSS $=$ Shapiro Tourette Syndrome Severity Scale

$\mathrm{THC}=$ tetrahydrocannabinol 